\title{
ODF Calculation by Series Expansion from Incompletely Measured Pole Figures Using the Positivity Condition Part I-Cubic Crystal Symmetry
}

\author{
M. DAHMS* and H. J. BUNGE \\ Institut für Metallkunde und Metallphysik der TU Clausthal, Großer Bruch 23, \\ D-3392 Clausthal-Zellerfeld, FRG
}

(Received 29 July 1987)

The calculation of orientation distribution functions (ODF) from incomplete pole figures can be carried out by an iterative procedure taking into account the positivity condition for all pole figures. This method strongly reduces instabilities which may occasionally occur in other methods.

KEY WORDS: ODF-analysis, iterative procedure, positivity condition, incomplete pole figures, cubic extrapolation, pole figure superposition, intermetallic compounds.

\section{INTRODUCTION}

Many methods have recently been presented to calculate a polycrystalline material's ODF from incompletely measured pole figures using series expansion (Bunge (1969), Pospiech and Jura (1974), Morris (1975), Kern and Bergmann (1978), Van Houtte (1980, 1984), Humbert and Bergmann (1980), Liang et al. (1981, 1983), Dahms and Bunge (1986a)). All these methods use the solution of a

${ }^{*}$ Now: GkSS Forschungszentrum Geesthacht; Postfach 1160; D-2054 Geesthacht. 
least squares problem, where the positivity condition

$$
P_{h k l}(\alpha \beta) \geq 0
$$

for all pole figures is generally not fulfilled particularly in the unmeasured range of the pole figures. In these methods the solution of the least-squares problem fits the measured range of the pole figures well but may lead to instabilities in the unmeasured range. It is the aim of this paper to show that it is possible to introduce the condition, Eq. (1), to standard routines without taking recourse to Van Houtte's (1983) quadratic method which requires extensive memory and computation time.

\section{Mathematical fundamentals}

In the harmonic method, the coefficients $F_{l}^{v}(h k l)$ of a pole figure are related to the coefficients $C_{l}^{\mu \nu}$ of the ODF by

$$
F_{l}^{v}(h k l)=\frac{4 \pi}{2 l+1} \sum_{\mu=1}^{M(l)} C_{l}^{\mu v} \dot{k_{l}^{\mu}}(h k l)
$$

where $\dot{k_{l}^{\mu}}(h k l)$ are spherical surface harmonics satisfying crystal symmetry.

The pole density values $P_{h k l}(\alpha \beta)$ of the corresponding pole figures can be calculated from

$$
P_{h k l}(\alpha \beta)=\sum_{l=0}^{L} \sum_{v=1}^{N(l)} F_{l}^{v}(h k l) \dot{k}_{l}^{v}(\alpha \beta)
$$

where $\dot{k}_{l}^{\nu}(\alpha \beta)$ are spherical surface harmonics satisfying sample symmetry.

In the case of completely measured pole figures, $F_{l}^{v}(h k l)$ can be calculated from

$$
F_{l}^{v}(h k l)=\int_{\alpha=0}^{\pi} \int_{\beta=0}^{2 \pi} P_{h k l}(\alpha \beta) \dot{k}_{l}^{* v}(\alpha \beta) \sin \alpha d \alpha d \beta
$$

where ${ }^{*}$ denotes the complex conjugate quantity.

The normalized pole density $P_{h k l}(\alpha \beta)$ cannot be measured directly. It must be determined from the measured intensity $I_{h k l}(\alpha \beta)$ using the relation

$$
P_{h k l}(\alpha \beta)=N_{h k l} \cdot I_{h k l}(\alpha \beta)
$$


where $N_{h k l}$ can be determined from the normalization condition $F_{0}^{1}=\sqrt{4 \pi}$.

Thus, for completely measured pole figures, Eq. (2) is an overdetermined system of linear equations because normally more than $M(l)$ pole figures are measured. This problem may be solved using a least squares method

$$
\sum_{i} w_{i}\left[F_{l}^{v}\left(h k l_{i}\right)-\frac{4 \pi}{2 l+1} \sum_{\mu=1}^{M(l)} C_{l}^{\mu v} \dot{k}_{l}^{\mu}\left(h k l_{i}\right)\right]^{2}=\min
$$

with $w_{i}$ as a weight factor.

The so determined $C_{l}^{\mu \nu}$ do not necessarily fulfill the positivity condition, Eq. (1), but normally this doesn't introduce serious errors, if pole figures with low indices are chosen for the ODF analysis.

In the case of incompletely measured pole figures, the $F_{l}^{v}$ cannot be calculated from the measured pole figures directly. With Eq. (5), Eq. (4) can be split into known and unknown parts:

$$
\begin{aligned}
F_{l}^{v}(h k l)= & 2 \cdot \int_{\alpha=0}^{\alpha_{\max }} \int_{\beta=0}^{2 \pi} N_{h k l} I_{h k l}(\alpha \beta) \dot{k}_{l}^{v}(\alpha \beta) \sin \alpha d \alpha d \beta \\
& +2 \int_{\alpha=\alpha_{\max }}^{\pi / 2} \int_{\beta=0}^{2 \pi} P_{h k l}(\alpha \beta) \dot{k}_{l}^{v}(\alpha \beta) \sin \alpha d \alpha d \beta
\end{aligned}
$$

In the case of cubic crystal symmetry, the $P_{h k l}(\alpha \beta)$ in the unknown part can be approximated by a cubic $\beta$-independent polynomial. We set in the unmeasured range:

$$
P_{h k l}(\alpha \beta) \sin \alpha=A+B \alpha+C \alpha^{2}+D \alpha^{3}=W_{h k l}(\alpha)
$$

the coefficients of which are obtained from boundary conditions at $\alpha=\alpha_{\max }$ and $\alpha=\pi / 2$ and the condition that $F_{2}^{1}(h k l)=0$. Details of this procedure were given in an earlier paper (Dahms and Bunge (1986a)). Using Eq. (8) as a first approximation for the unmeasured range of the pole figure, the second part of Eq. (7) becomes zero for any $v \neq 1$.

Thus, the pole figure can be normalized in a first approximation, and with Eq. (6), first-order $C_{l}^{\mu \nu}$ can be calculated. With these coefficients the input pole figures can be recalculated in the complete range using Eqs. (2) and (3). In order to fulfill Eq. (1), all so obtained negative pole figure values are set to zero. 
A second order estimation of the unknown part of Eq. (7) is thus obtained, leading to a second order estimation of the $F_{l}^{v}(h k l)$. With Eq. (6), the $C_{l}^{\mu \nu}$ can be calculated again and a loop is defined, which can be run through as often as desired. There is no risk of divergence, because no negative values are allowed according to Eq. (1) and the maximum positive values are limited by the normalization. The flux diagram for this procedure can be seen in Figure 1.

It is also possible to introduce additional unmeasured pole figures after the first loop. They can be treated in the same way as the unmeasured ranges of the measured pole figures. Also in these pole figures, the condition Eq. (1) is enforced by setting all negative values to zero.

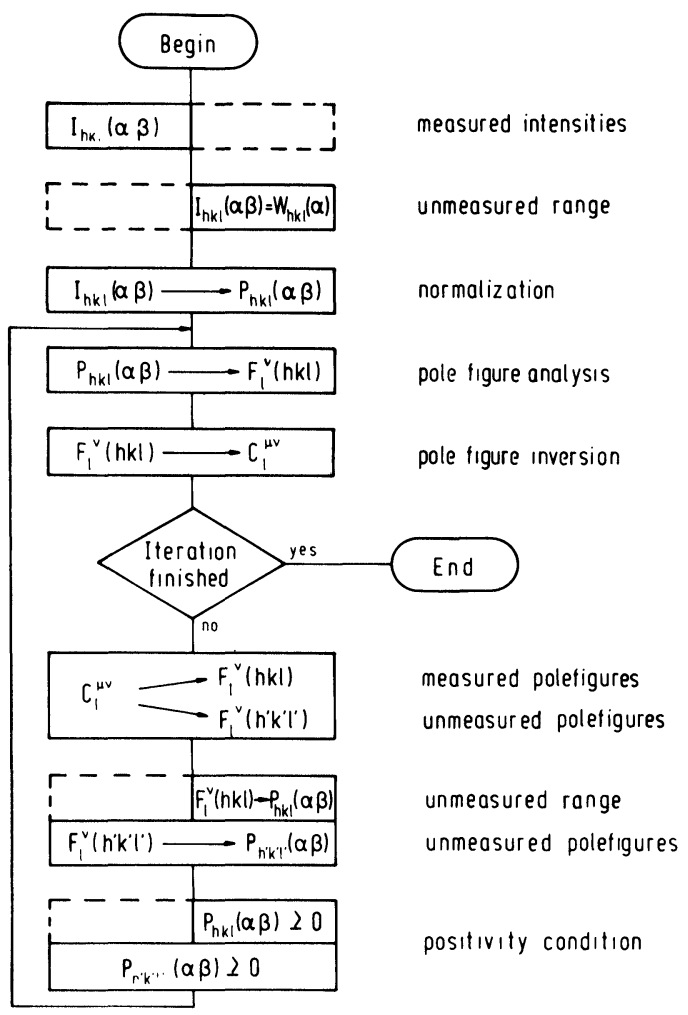

Figure 1 Flux diagram of the iterative procedure. 
In the case of coincident pole figures and unknown overlapping factors $q_{i}$, it is also possible to calculate the overlapping factors in the same loop using the method presented in another paper (Dahms and Bunge (1986b)).

\section{RESULTS}

In order to test the proposed method, one synthetic and two real textures of cubic crystal and orthorhombic sample symmetry were used.

The synthetic texture was a cube texture with a spread of $12.5^{\circ}$. Theoretical pole figures were calculated as described by Dahms and Bunge (1986a) in steps of $\Delta \alpha=5^{\circ}$ and $\Delta \beta=3.6^{\circ}$ up to $\alpha_{\max }=70^{\circ}$ in the approximation $L=34$. The pole figures (100), (110), (111) and (311) were used.

The first real texture was the texture of a tensile sample of a cold rolled and recrystallized ferritic steel after $20 \%$ elongation. Here, the (110), (200) and (211) pole figures were measured using $\operatorname{Co}(K \alpha)$ radiation with the same steps as the theoretical texture up to $\alpha_{\max }=70^{\circ}$. Only three pole figures could be measured because the maximum Bragg angle of the goniometer was $50^{\circ}$.

The second real texture was the texture of a sample of the intermetallic phase $\mathrm{Cu}_{9} \mathrm{Al}_{4}$ which was hot compressed at $735^{\circ} \mathrm{C}$ to $\varepsilon_{h}=2.9$ with ratio $\varepsilon_{l} / \varepsilon_{w}=0.2$. In this case, the $(411) /(330)$, (442)/(600), (444), (721)/(633)/(552) and (741) pole figures were measured using $\operatorname{Co}(K \alpha)$ radiation. Details of the measurements for this material are discussed in another paper (Dahms and Bunge (1986b)).

\section{Cube texture}

From the four synthetic pole figures, $C_{l}^{\mu v}$ for even $l$ up to $L=34$ were calculated using the iterative procedure described above. For comparison, also the non-iterative standard ODF routine for incomplete pole figures (Dahms and Bunge (1986a)), which solves a least-squares problem over the measured range of the pole figures with estimated normalization factors proposed by Bunge (1969), was used. Figure 2 shows the mean values of the $C_{l}^{\mu v}$ for every $l$ for 


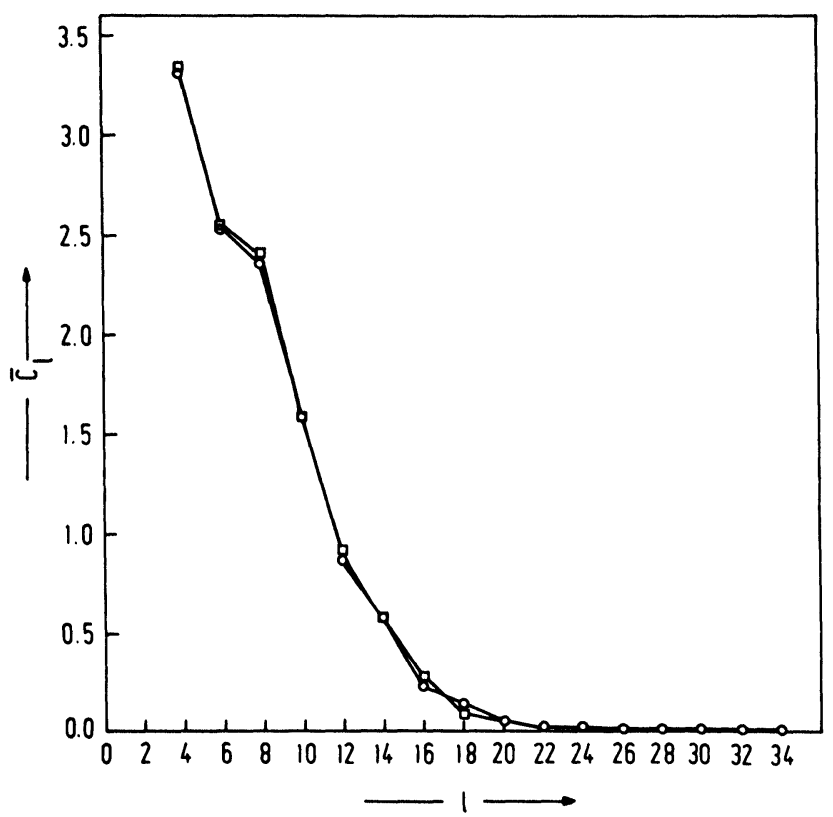

Figure 2 Mean absolute values of the $C_{l}^{\mu \nu}$ vs. degree of series expansion $l$ for a cube texture with $\omega_{0}=12.5^{\circ}$ : $(\bigcirc)$ iterative; $(\square)$ non-iterative procedure.

both methods. It can be seen that the difference is very small. Table 1 shows the maximum value of the ODF at the cube position for both methods compared to the ideal one, which is calculated directly from the original $C_{l}^{\mu \nu}$.

It can be seen that the iterative procedure results in a slight smoothening of the calculated texture. This is also evident looking to the texture index $J=16.1$ in the iterative procedure compared to 16.4 in the non-iterative.

Table 1 Maximum values $f_{\max }$ of the ODF for a cube texture with $\omega_{0}=12.5^{\circ}$

$\begin{array}{ll}\text { ideal } & 44.0 \\ \text { non-iterative } & 43.5 \\ \text { iterative } & 42.4\end{array}$




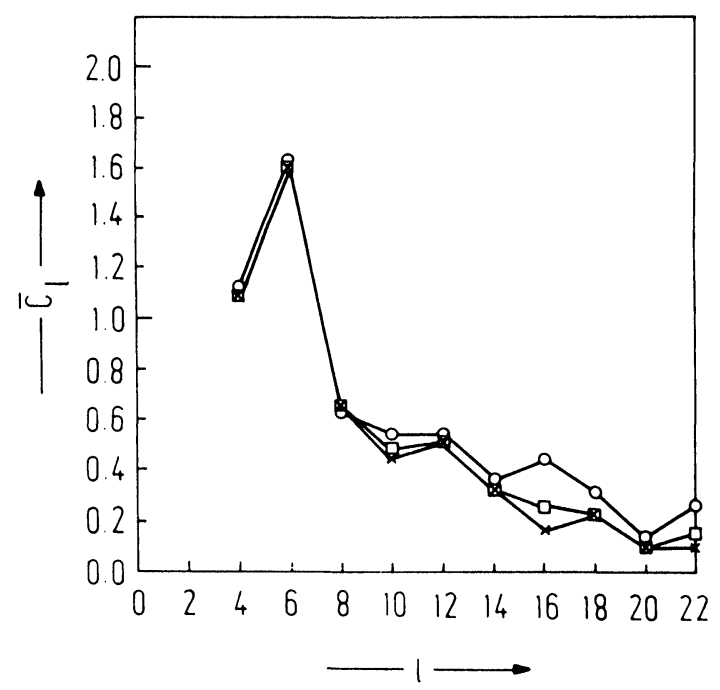

Figure 3 Mean absolute values of the $C_{l}^{\mu v}$ vs. degree of series expansion $l$ of the texture of a ferritic steel: $(O)$ non-iterative; $(\square)$ iterative procedure; $(\times)$ iterative procedure with additional (111) pole figure.

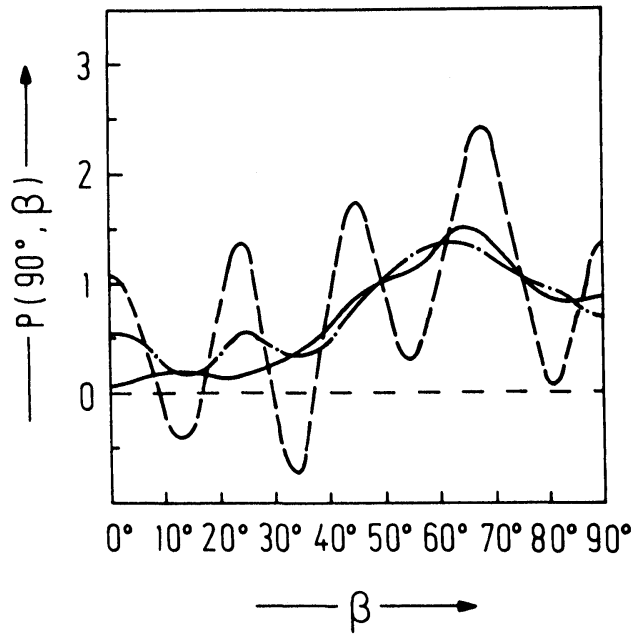

Figure $4 \alpha=90^{\circ}$ section of the unmeasured (111) pole figure of the texture of Figure 3: $(---)$ non-iterative; $(-\cdot-)$ iterative procedure; $(-)$ iterative procedure with additional (111) pole figure. 


\section{Texture of a ferritic steel}

Here, the ODF was calculated up to $L=22$ using the iterative and the non-iterative procedure as well as the iterative procedure with an additional (111) pole figure. Figure 3 shows the $C$-mean for all cases. Obviously, up to $l=14$ there is no significant difference between the methods. At higher $l$, the iterative methods converge to lower values, whereas the standard routine does not lead to strictly convergent coefficients. This is due to the fact that three experimental error-containing pole figures measured up to $\alpha_{\max }=$

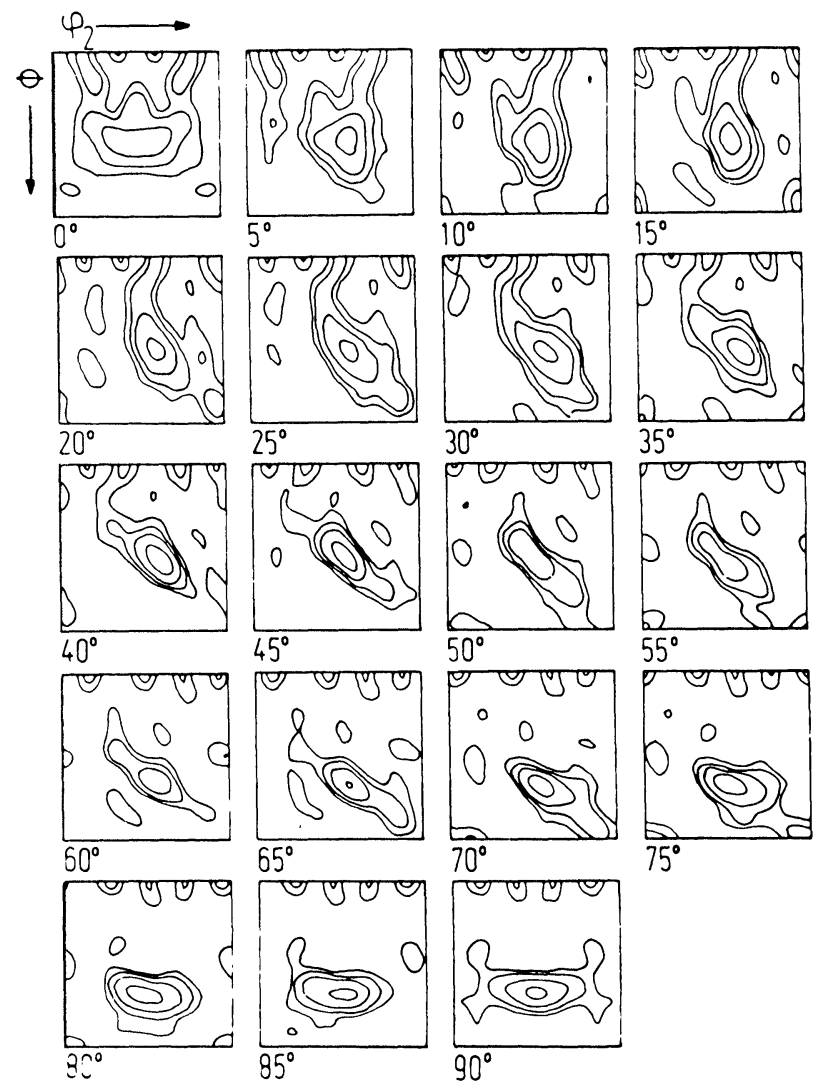

Figure 5a Complete ODF of the texture of Figure 3: non-iterative ( $L=23$, $\varphi_{1}=$ const., contour levels $1,2,4,8 \times$ random). 

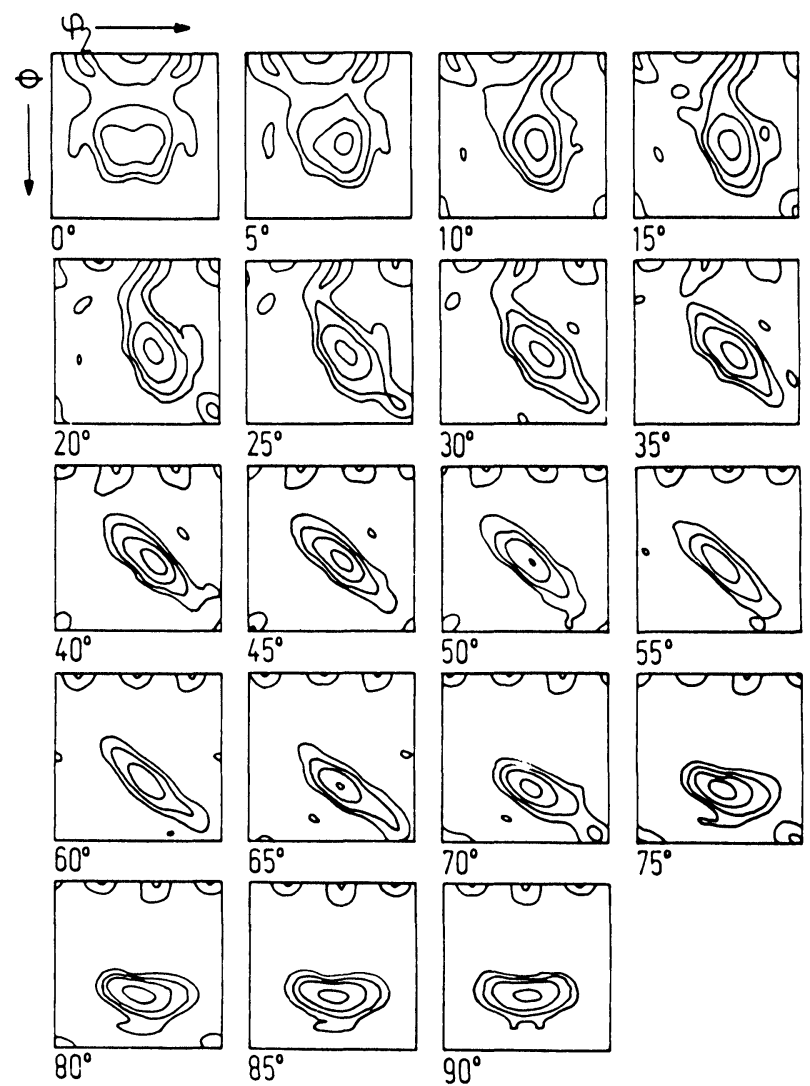

Figure 5b Complete ODF of the texture of Figure 3: iterative procedure ( $L=23$, $\varphi_{1}=$ const., contour levels $1,2,4,8 \times$ random).

$70^{\circ}$ normally do not contain enough information to calculate $C_{l}^{\mu v}$ up to $L=22$ with enough precision (Pospiech and Jura (1974)). The positivity condition, however, introduces additional information which leads to stable results.

In Figure 4, the $P_{h k l}$ for $\alpha=90^{\circ}$ of the unmeasured (111) pole figures recalculated from the $C_{l}^{\mu \nu}$ of all methods are shown. It can be seen that the standard routine for incomplete figures leads to unstable oscillating results, whereas the iterative methods, using the positivity condition in the measured pole figures, lead to stable 


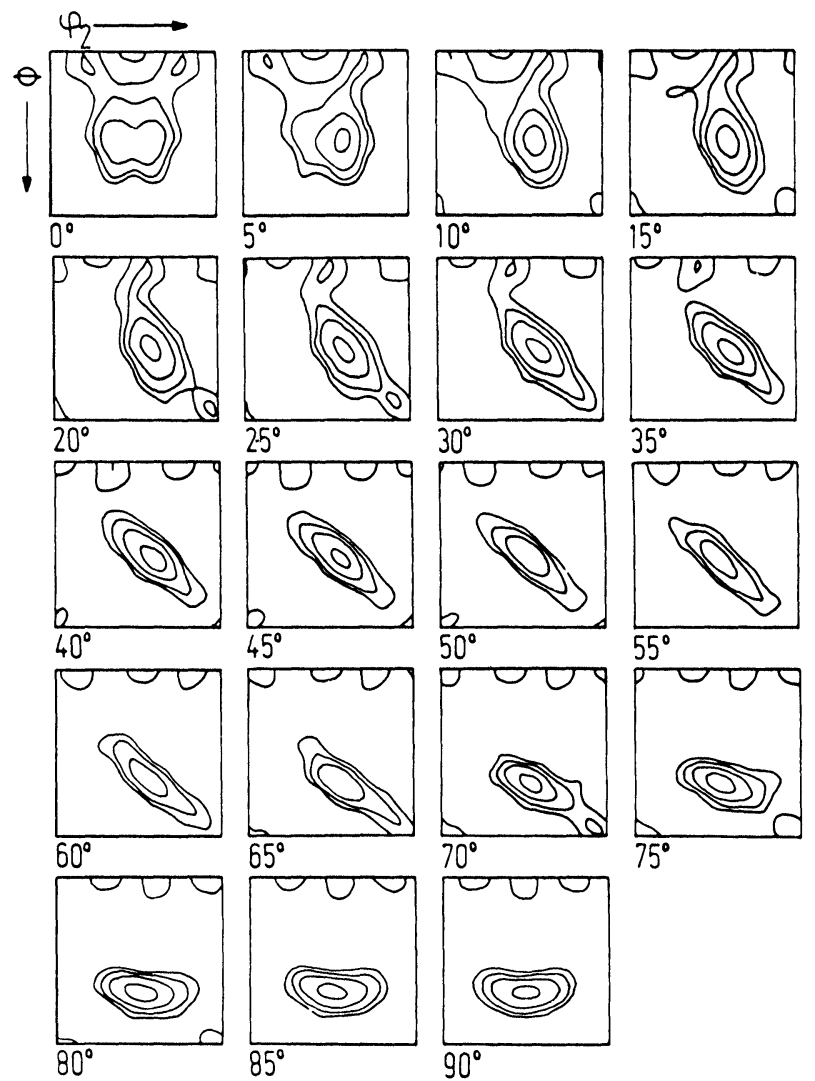

Figure 5c Complete ODF of the texture of Figure 3: iterative procedure with (111) pole figure $\left(L=23, \varphi_{1}=\right.$ const., contour levels $1,2,4,8 \times$ random $)$.

non-oscillating calculated pole figures, even in the unmeasured ranges. The iteration using an additional (111)-pole figure leads to the most stable recalculated pole figure with a maximum value at $\beta=63^{\circ}$.

Figures 5a-c show the resulting ODFs in the projection $\varphi_{1}=$ const. The $C_{l}^{\mu \nu}$ for odd $l$ were calculated using the zero-range method as described for example by Lee (1985) up to $L=23$. Oscillations introduced by the standard routine can also be seen here, which disappear in the iterative methods such that the resulting ODFs are clarified. The "weighted sum of negative intensities," a value proportional to the integral over the negative 
ranges, is -192 in the case of the iterative method and -452 in the case of the standard routine. The best results are obtained with the additional (111) pole figure, where this value decreases to -83 . Thus, the iterative method with the (111) pole figure leads to the best results, if there are only three incomplete pole figures available. This fact has been proven in several other calculations.

\section{Texture of an intermetallic compound}

Here, the ODF was calculated up to $L=34$ because of the sharp measured pole figures. The ODF was again calculated using the standard and iterative methods. In both cases, overlapping factors were considered to be known according to the ASTM Data Card 24-4A. As a third possibility, the overlapping factors of the coincident pole figures were treated as unknown and were calculated together with the $C_{l}^{\mu v}$ during the iterative procedure according to Dahms and Bunge (1986b).

Mean values of the $C_{l}^{\mu \nu}$ are shown in Figure 6. In all cases, they

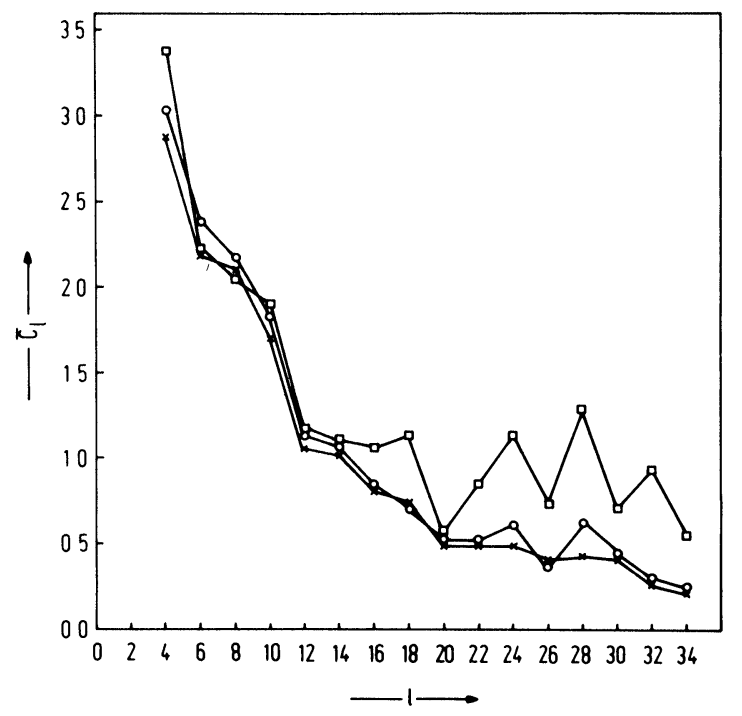

Figure 6 Mean absolute values of the $C_{l}^{\mu \nu}$ vs. degree of series expansion $l$ of the texture of the intermetallic phase $\mathrm{Cu}_{9} \mathrm{Al}_{4}:(\square)$ non-iterative; $(O)$ iterative procedure; $(\times)$ iterative procedure with iteratively determined $q_{i}$. 
tend to decrease with increasing $l$, but the non-iterative procedure does not converge at higher degrees of series expansion. The weighted sum of negative intensities in the ODF with $l_{\text {odd }}$ up to $l=23$ is thus -5967 in the case of the standard routine compared to -1808 in the case of the iterative procedure and -1507 for the third method.

Figures $7 \mathrm{a}$ and $\mathrm{b}$ show the corresponding ODFs in sections of $\varphi_{2}=$ const. They exhibit nearly ideal cube textures with an inhomogenous spread for the iterative procedures. The standard

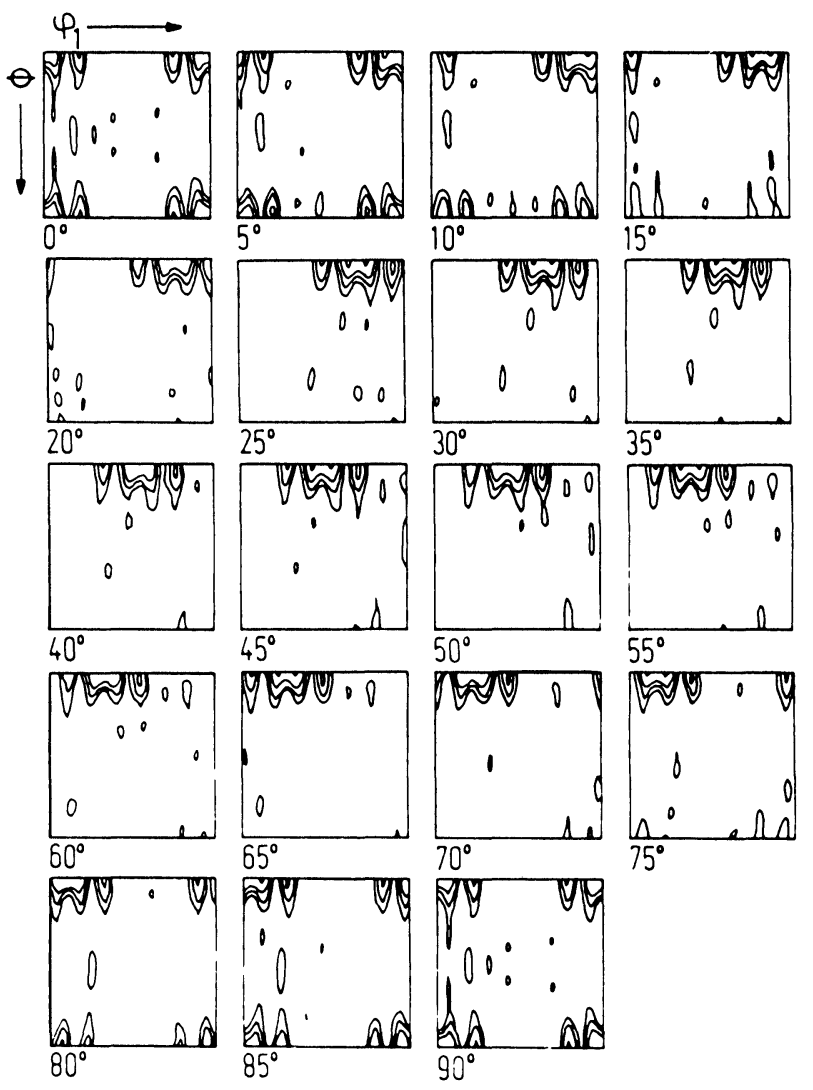

Figure 7a Complete ODF of the texture Figure 6: non-iterative ( $L_{\text {even }}=34$, $L_{\text {odd }}=23, \varphi_{2}=$ const., contour levels $25,35,50,70 \times$ random). 


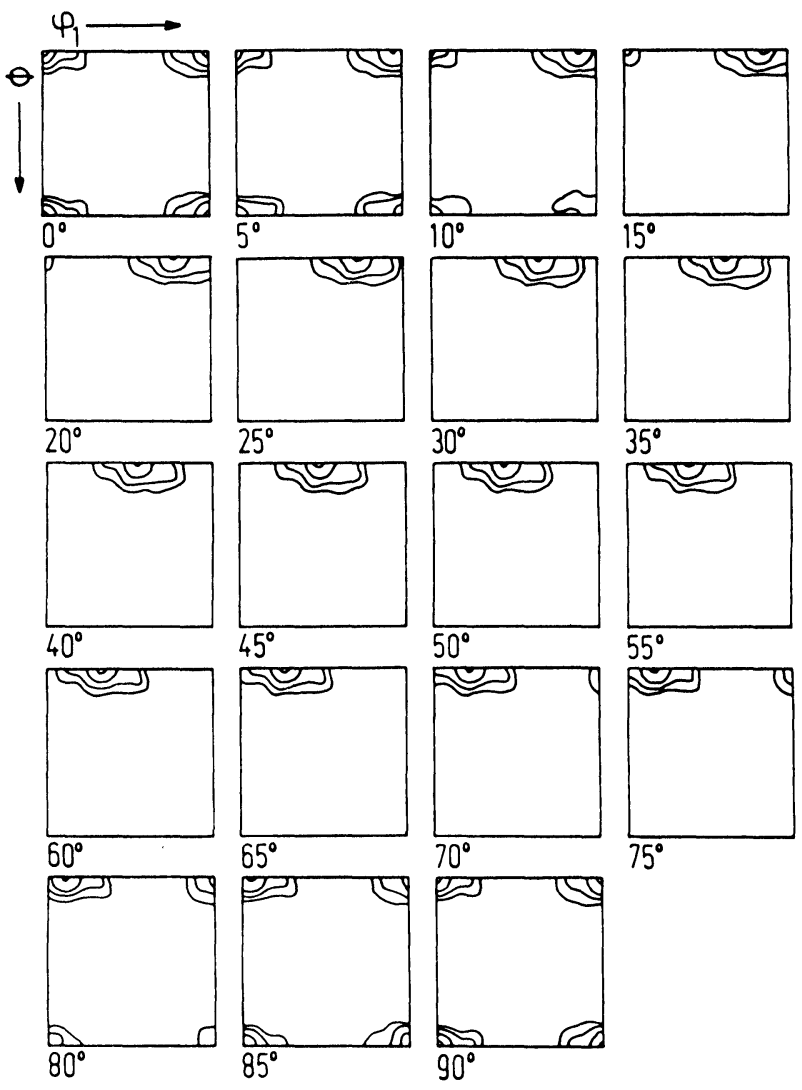

Figure 7b Complete ODF of the texture Figure 6: iterative procedure $\left(L_{\text {even }}=34\right.$, $L_{\text {odd }}=23, \varphi_{2}=$ const., contour levels $25,35,50,70 \times$ random).

routine leads to an oscillating ODF which has no physical meaning. The oscillations are due to the large values of the high-order $C_{l}^{\mu \nu}$. Oscillations do appear also in the iterative procedures, as can be seen in Figure 8 which shows the $\alpha=90^{\circ}$ section of the recalculated (100) pole figures, but they are tolerably damped. The oscillations in the iterative procedures are due to the fact that the (100) and (110) reflections are not measured separately and the (444) reflection has an extremely low peak-to-background ratio as was shown in a previous paper (Dahms and Bunge (1986b)). 


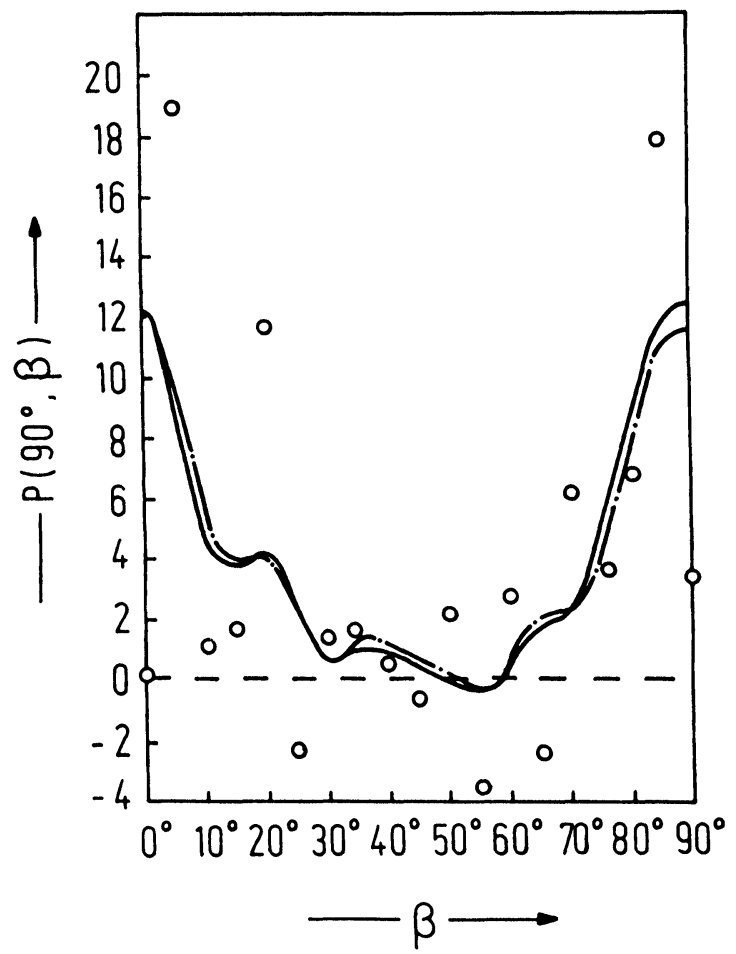

Figure $8 \alpha=90^{\circ}$ section of the (100) pole figure of the texture of Figure 6: $(O)$ non-iterative; $(-)$ iterative procedure; $(-\cdot-)$ iterative procedure with iteratively determined $q_{i}$.

\section{CONCLUSIONS}

The iterative method described here including the positivity condition of all pole figures allows the calculation of the ODF to higher degrees of series expansion $L$ with less pole figures than the standard method, which only solves the least-squares problem on the measured range of the pole figures. The resultant smoothing of the "true" texture seems tolerable. In cases where the measurement is more precise than the smoothing effect, the use of the standard method may be indicated. A positive side effect of the present method is, that it essentially relies on the standard program system for complete pole figures. 


\section{References}

Bunge, H. J. (1969). Mathematische Methoden der Texturanalyse. Akademie Verlag, Berlin.

Bunge, H. J. (1982). Texture Analysis in Materials Science. Butterworths Publ., London.

Dahms, M. and Bunge, H. J. (1986). Calculation of the Normalization Factor of Incomplete Pole Figures by Cubic Extrapolation. Textures and Microstructures 6, 167-179.

Dahms, M. and Bunge, H. J. (1986). Texture Determination in Intermetallic Phases. In: Experimental Methods of Texture Analysis. Ed: H. J. Bunge. DGM Informations gesellschaft Oberursel, 371-378.

Dahms, M. and Bunge, H. J. (1987). Texture Analysis Using Totally and Partially Coincident Pole Figures. In: Theoretical Methods of Texture Analysis. Ed: H. J. Bunge. DGM Informationsgesellschaft, Oberursel, in print.

Humbert, M. and Bergmann, H. W. (1980). A Determination of the Normalization Factor and its Application to the Incomplete Pole Figure Method. J. Appl. Cryst. 13, 500-504.

Kern, R. and Bergmann, H. W. (1978). A Simple Way for Calculation ODF from Incomplete Pole Figures. In: Textures of Materials, Proc. ICOTOM 5. Ed.: G. Gottstein and K. Lücke, Springer Verlag, Berlin, 139-147.

Lee, H. P. (1985). Texturanalyse unter Anwendung der Nullbereichsmethode. Thesis Clausthal.

Liang, Z., Xu, J. and Wang, F. (1981). Determination of ODF of Polycrystalline Materials from Incomplete Pole Figures. Proc. ICOTOM 6, Tokyo, 1259-1264.

Liang, Z., Xu, J. and Wang, F. (1983). Orientation Distribution Analysis of Hexagonal Materials from Incomplete Pole Figures. Mat. Sci. Eng. 60, 59-63.

Morris, P. R. (1975). Crystallite Orientation Analysis from Incomplete Pole Figures. Adv. X-ray Anal. 18, 514-534.

Pospiech, J. and Jura, J. (1974). Determination of the Orientation Distribution Function from Incomplete Pole Figures. Z. Metallkunde 65, 324-330.

Van Houtte, P. (1980). A Method of Orientation Distribution Function Analysis from Incomplete Pole Figures Normalized by an Iterative Method. Mat. Sci. Eng. 43, 7-11.

Van Houtte, P. (1983). The Use of a Quadratic Form for the Determination of Non-Negative Texture Functions. Textures and Microstructures 6, 1-19.

Van Houtte, P. (1984). A New Method for the Determination of Texture Functions from Incomplete Pole Figures-Comparison with Other Methods. Textures and Microstructures 6, 137-161. 